\title{
REVIEW OF WEB-BASED INTELLIGENT BUILDING SYSTEM
}

\author{
Sayali P. Patil ${ }^{1}$ and Prof. Sanjeev N. Jain ${ }^{2}$
}

\begin{abstract}
The Internet of Things is what the so much talked upcoming possibility and challenges for the internet. To obtain flexibility and scalability, this expansion of web architecture to a completely new domain of constrained wireless network and devices will play a very important role. The intention of this paper is to shed some light on the introduction of web service based automatic control system. It also proposed renewable energy, solar energy for improving the working capacity and distributed control centralized management system of buildings. The Measured energy performance source is necessary to demotic efforts to improve building efficiency, as proved in new benchmarking mandates, and with the increasing body of work that signalizes the value of stable monitoring and energy acquaintance feedback. These building energy information systems designed intent to endow water distribution, energy, air-conditioning system, hybrid physics-based and data-driven approach to optimal control of building cooling/heating systems.
\end{abstract}

Keywords - Building Automation; Web Service; Energy Information System; Solar Energy; Air Conditioning.

\section{INTRODUCTION}

In the realm of Automation Technology, Intelligent Building is taking place as a fresh focus. In ameliorating the energy function in building the idea if the intelligent building is useful. The real-time framework consists monitoring software, data acquisition hardware. To store, analyze and display buildings, real time data communication system is applied. This method bestows hourly entire building electric data which is the web accessible with analytical and graphical efficiency. With the promotion of real-time system, we can alleviate energy consumption in building for certain causes. i.e. Energy saving, Decrease maintenance expenditure, Detract expensive visits, developed safety.

The rest of the paper is organized as follows. Web Services and Web Architecture are explained in section 1.1 and 1.2. Literature Surveys, Recent Search and Applications are presented in section II, III and IV. Concluding remarks are given in section V.

\subsection{WEB SERVICES}

To grasp how to expand the web to suppressed networks and device, it is also important to understand the core architecture of the web. A recent application transfer protocol designed for

\footnotetext{
${ }^{1} P G$ Student, Department of Electronics Engineering, SSVPS BSD's COE, Dhule, Maharashtra, India

${ }^{2}$ Associate Professor, Department of Electronics Engineering, SSVPS BSD's COE, Dhule, Maharashtra, India
} 
cognizing embedded web services was developed by the RESTful environment group. Due to the Internet of Things (IoT), there will be plenty of challenges in the front of the internet and communication engineers because internet growth is from millions to billions. Figure 1. Show ring model of Internet of Things. The Third ring on the internet which is Internet of Things consists of recently embedded application such as routers, servers and quickly growing fringe internet consists of smart phones, personal computers. All above applications add total probabilities and challenges.

Web Services is a program running on the remote device, also known as a web server which using a transport and vendor neutral protocol exposes it to the web. It consists of two communicating entities Producer and Consumer i.e. Web server and Client. A more sophisticated form also involves registry acting as a third party context as to which web service endow which service at what address.

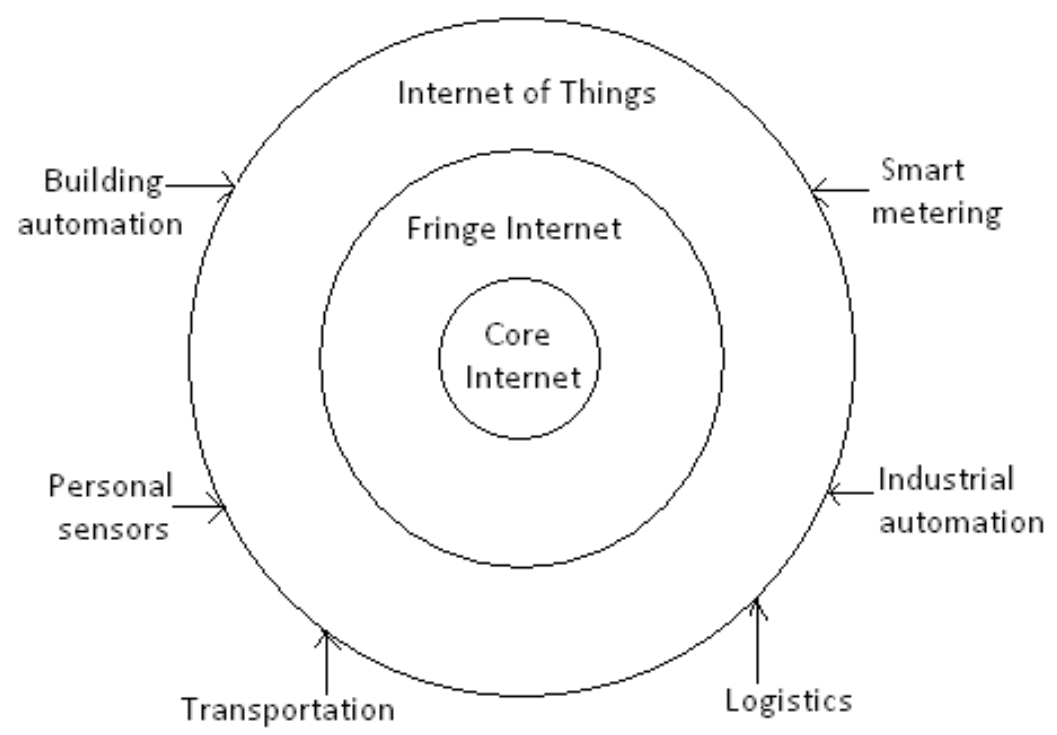

Fig 1.Internat of Things

\subsection{WEB ARCHITECTURE}

Figure 2 is the Server Controlled Resources they are accessed by clients in a synchronous request/response manner using methods such as GET, PUT, POST and DELETE of HTTP. Resource state permits for the Catching, Proxy, and Redirection of Requests and Response. Web Resources may contain links to other resources and to build a distributed web between Internet endpoints, resulting in highly scalable and flexible architecture. This web architecture is commonly known as Representational State Transfer (REST). Though today HTTP is solely being used for tampering web Resources, REST can also be used with other application protocol. 


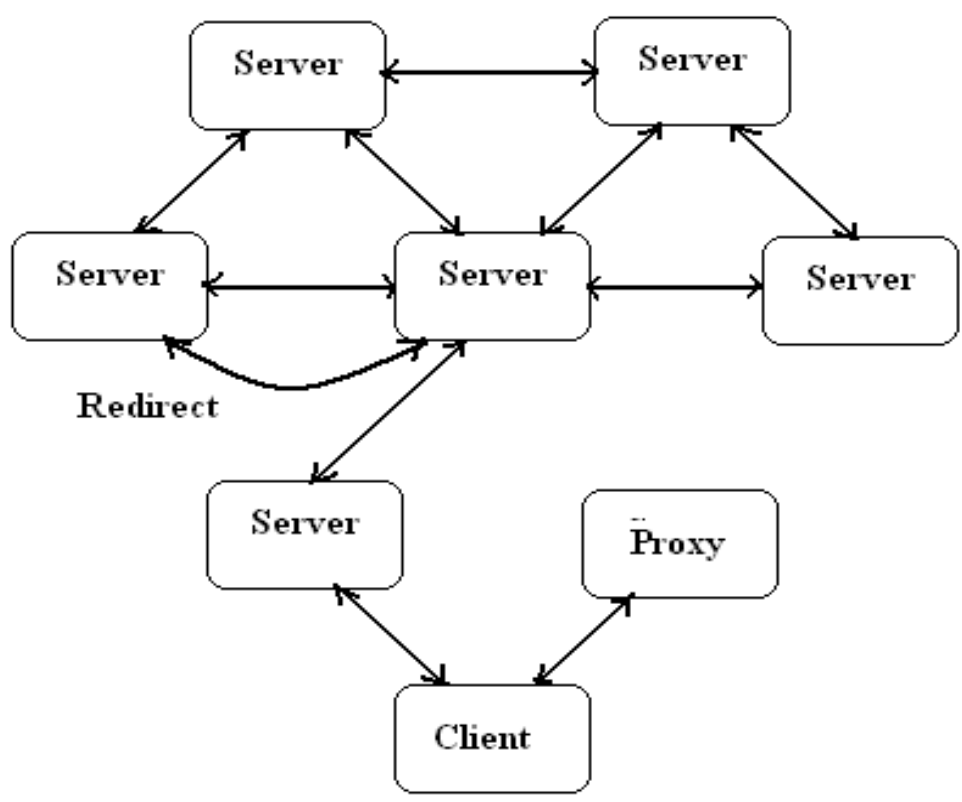

Fig 2. Web Architecture.

\section{LITERATURE SURVEY}

2.1 The Method I: Network Communication Of the System Implemented Based on TCP/IP Protocol.

2.1.1 For improving the operating efficiency and realize the distributed control and centralized management of buildings. This paper introduced an automatic control system based on Advantech web access for intelligent buildings and renewable energy solar energy source. As the communication network, this system uses $485 \mathrm{Net}$ and Ethernet. Monitoring and Controlling of equipment are implemented on an ADAM-5510/TCP controller and ADAM-5000/TCP controller of Advantech. Web access configuration software accomplished the device management and control of the building. Results show that the system design based on Web access configuration software and ADAM controller is suitable, and research on the solar energy in automation, building applications is a justified job [1].

To obtain full automation, centralized data management, and information controls the building monitoring system links computer science,technology, Internet technology and programmable, control technology. Using configuration software Web access application the building control system provides complete automatic adjustment, automatic monitoring, automatic alarm function and self-diagnosis, etc. All these advantages cause the reduction of the amount of equipment operates and operations cost and improve supervision efficiency of the intelligent building [1].

2.1.2 The technique has been existent for home energy management and control, leveraging the Internet of Things protocol stack, including emerging IETF standards such as CoAP, RPL, and 6LoWPAN Protocols. The open standards founded outlook enables seamless integration of the equipment's at home to the present internet infrastructure, making remote retrospect, control and data set over the web. Further, we enable IPv6 connectivity to the energy meter direction to a superior integration of the source material management systems of the subservience unlike some 
of the present possessor solutions. To produce electricity on PV panel for DC appliances different from the main $230 \mathrm{~V}$ AC supply and IPv6 network connected to the system. The twoway communication between the utility of the system and the user over the internet by using wireless node that based on CC2538 System-on-Chip and interfaced with an energy meter [2].

The real-time execution of home energy management based on internet protocols for monitoring and controlling the electricity and experiment. The prototype execution of a 6LoWPAN protocol of allowed wireless sensor network by using CoAP at the application layer. The operable electricity requirement in buildings cannot be met perfectly without the use of renewable sources of energy. This implemented the design of a system. Successfully exhibit's a path of leading power from both energy sources. i.e. renewable and non-renewable operating in an equal. We can reduce dependency on AC power, hence reducing costs and saving energy using the application powered. To scale energy consumption for the intention of calculating the bill (use of energy) in real time using the power meter, will be available to both i.e. appropriator and user. Consumers can also control the applications from any destination through an internet. The main purpose of this automatic experiment timing based switching of heavy loads can be an enormously helpful path of protecting energy and removing human intervention [2].

2.1.3 Water distribution system offers important challenges for structural monitoring. They include a complicated network of pipelines buried underground, which are relatively inaccessible. For providing clean drinking water to the general public maintaining integrity of these networks is very important. To facilitate the efficient management and operation there is need online-watering of water distribution systems. Whenever a pipe failure occurs, it is important to discover and localize them. It is also important to identify the areas of the distribution network, which are more likely to be susceptible to structural failure. These capabilities are very important to reduce the time taken to identify and repair failures [3].

The water wise pressure data collection has been used to designate subscription of pressure transients from a range of normal recurring operations, routing pumping, valve operations. By building a classification system of the transients, unusual subscription from undocumented sources are more readily identified and this provides a liberal of promises approach for remote detection of more slowly evolving events. The water wise monitoring system supply impassable data within the distribution network, which can enable optimization pumping costs. The ongoing research aims to develop a more reliable understanding to detect water quality anomalies [3].

2.1.4 As energy consumption is widely recognized the primary defense of wireless networked devices, energy harvesting emerges as a liberal of promises path of powering the Internet of Things. Energy harvesting introduces spatial and changeable uncertainty in the availability of energy. The main intention of this paper is to design and execution of the MAC layer of wireless embedded systems that are enabled by energy harvesting. This system provides novel protocol features and practical experiences to designers of user electronics who opt for tailoring their own protocol denouement instead of using standards [4].

A liberal of promises denouement for enabling sensing and IoT enabling technologies is constituted by energy harvesting. It introduces spatial and short-term uncertainty in the MAC layer of communications which controls the duty cycles. It aims to tackle the defiances of energy harvesting communications. Along with other novel protocol features the individual control of 
the duty cycles, gives to a sustainable work and to the overall energy efficiency of the communication. This evaluation exhibits long term sustainable operations and offers configuration parameters which allow the system designers to control the trade-off between sustainable flow capacity and link delay the experiments show that opportunistic forwarding and altruistic back off improve the energy efficiency of the link. Compared to standard solutions the efficiency of the link to improve up to $32 \%$ and $26 \%$ Respectively [4].

2.2 Method II: Air-conditioning optimization control system is designed founded in Lab VIEW graphical programming software.

2.2.1 Optimal automatic features of the air-conditioning system are calibrated in the article. The designed and developed based on Lab VIEW graphical programming software, and cold water temperature, the cold water flow, and cooling water flow can be integrated controls for reducing energy consumption. The application demonstrates that the optimal control management system of central air-conditioning can achieve static and reliable operation, and also energy saving effect. The central air-conditioning optimization control management system which is based on research of air-conditioning system optimization operation strategy has been designed and developed it had been successfully applied in the energy saving renovation project in a government office building [5].

The result shows that the management system can achieve a static and reliable operation process and achieves an excellent energy saving effect. Central air-conditioning optimization control management system makes full use of the original control system in which "a single pump frequency conversion, plus power frequency" parallel operation mode has been adopted, and it can realize cold and cooling water system genuine variable flow operation by only two frequency converters [5].

2.2.2 These projects works concatenate a physical-based model with a data-driven time-series model to forecast and optimally operate building energy. Physical description of the building is partly captured by an accumulation of zonal energy balance equations with parameters estimated using a Least Squares Estimation (LSE) technique and data at the beginning germinated from the energy-plus building model. A generalized Cochran-Orcutt computation technique is adopted to represent the data produced from these simulations. The included forecast model is then used in model predictive control (MPC). A framework to manage heating and cooling set points. This work is stimulated by the practical limitations of simulation-based optimization. Once the forecast model is placed capturing sufficient statistical variability and physical behavior of the system, there is no need to run energy plays in the optimization routine [6].

Another probable reformation for the proposed control strategy is to employ more accomplishes methods for the MMP problem. The Utility Function Views, for illustration, can be used instead of the weighted metrical method to complete the lowest and highest desirable values of the objective functions [6].

\subsection{Method III: Implementation of Raspberry Pi Operating System.}

2.3.1 The project offers potential execution for IoT used for monitoring and controlling the home loads via the World Wide Web. The whole system uses the portable devices as a user interface. Through an Internet gateway, they can communicate with the home automation network, by 
means of low-power communication protocols like Wi-Fi, Zigbee. etc. This project objective is to control home applications via smartphones by using Wi-Fi as the communication protocol and raspberry pi as the server. Using this system the user will move directly through a web-based interface over the web, whereas home appliances like door lock, lights, and fan are remotely controlled via a website. The one more feature of this system is to protect fireplace accidents. Whenever it will detect smoke that time it will send an image to the smartphone and associates an alerting message. The server interfaced with relay hardware circuits which control the appliances running at home and the user can select the appropriate device by communicating with the server [7].

The system proposed the occurrence of a home monograph and security system exploitation using a raspberry pi and Internet of Things (IoT) technology. By this system endow a climbable and price dominant home automation system. This system dramatically cuts back the hazard of unauthorized entry in many places like labs, banks, hospitals etc. [7].

2.3.2 In this technology, we are reducing the standby strength. The indicated system consists of a ZigBee hub and server, an automatic standby strength cutoff outlet. When Power outlet is less than fixed value, then the energy consumption of the device is connected to the power and the strength outlet with ZigBee module cuts off the AC power. The focal hub of the ZigBee collects information from the power drains. Through the ZigBee segment can control the power channels. The user can monitor or control the present energy after sending the present state information to a server by using the HEMS user interface [8].

In this document, real-time smart power management system, flexible, a low cost, that can merely integrate and function with the home observing systems. This scheme is able to accomplish the remote monitoring and control of loads successfully [8].

2.4 Method IV: A neural network based multiple robot localization and mapping scheme.

2.4.1 A quadtree based data assembly scheme is introduced in this work. Whereby the mobile sink corporeally movements through every room and logical connections all separated subnetworks together. These schemes sequentially gather data from the monitoring environment and transmit the statement back to a base station. According to the sensor node information, a command can be given by the BS to the cleaning the robot to move to a specific place in the home environment. This scheme minimizes the data assembly tour length and time through competency allocation of data assembly areas. A calculated shortest path data assembly tour can efficiently be allocated to the robotic cleaner to complete the cleaning task within a minimum time period [9].

At last simulation, outcomes demonstrate that the introduced scheme can effectively allocate and control the purifying area of the robot vacuum cleaner in absentia any direct interface from the user. The performance of the introduced scheme is then condescending with a group of practical successional data assembly tours in a typical office [9].

2.4.2 The key ambition, mutually municipal managers plus reserved administrators aim of optimizing the office block power consumptions. The obtainable district energy organization methodology can be applied in the actual world for two purposes: 
- $\quad$ Energy wastes and prices are decreased using economic benefits for operators, residents, and communal supervisions [10].

- The power consumption is compact by giving that environmental also social profit. Upcoming exploration will consider the impression of alternative energy sources, in addition, will take the graphical and air-quality luxury into the description [10].

The existing approach that is created on the redistribution of penalties and booty amongst district structures guarantees a real-time energy cost saving and assures handler's thermal ease [10].

\section{RECENT SEARCH}

3.1 Method I: Design of Linux Infrared Remote Setup on Raspberry Pi and Time Synchronization Technique for CoAP- Based Automation System.

3.1.1 This paper proposes an approach to enhance old appliances and the controlling experience through an Internet of Things based Home appliance controlling system. Due to the sensors the Home appliances can be controlled environment condition trigger. The output of the sensor means sensor data are processed by single board computer and these data is delivered to a the mobile application through wireless connection. The result of implementation and experimentation shows the proposed system and platform can provide more Internet of Things application possibilities in daily life. Any control system to provide user-centered experience for home appliances controlling.

The main objective of this system is based on compatibility and usability needs. With the present platform more and more sensors can be connected and streamed data to the internet in the future with this system not only home appliances but also more devices and services will be involved [11].

3.1.2 The objectives of this design and implementation of an Ethernet-based intelligent automated system is conserved electrical energy using INTEL GALILEO $2^{\text {nd }}$ generation development board, which can be used in a large organization like a university or an office. This proposed system works on automation for avoiding human intervention.

In this scheme can also the available class infrastructure to minimize the cost criteria. It is monitored and controlled by the web server by using the Internet or the Intranet connectivity. To reduce the power consumption of the system by controlling the power based on the academic timetable of a university and also minimizes computation power [12].

3.1.3 The aim of the projects is to develop time synchronization techniques for low power sensor modules are highly demanded. The IETF standardized Constrained Application Protocol (CoAP) for sensor networks; it is also being widely adopted for home automation system by ETSI, OMA, and one M2M. This paper gives a lightweight time synchronization algorithm for Constrained Application Protocol (CoAP) based home automation networks. In this home automation system, the CoAP option field and a shim header are used to include time stamps. This proposed scheme works for both IP-based and Non-IP-based home automation. Accordingly, general protocols, like TPSN, RBS, RTSP and IEEE 1588. Experiments by several household devices with Non-IP communication Interface, the result of proposed technique gives an average error of $1 \mathrm{~ms}$ and a network overhead reduction of $17 \%$ when compared to the ideal NTP service. 
This proposed scheme uses CoAP instead of additional standard for time synchronization protocols. CoAP based time synchronization can be broadly applied, not only in the home automation system, but also in other applications such as disaster monitoring, urban monitoring, environmental monitoring, building and plant management etc. [13].

\section{APPLICATIONS}

1. Smart Home: The purpose behind product of smart home is for switching lights on mode $\mathrm{ON}$ and OFF. It switches Air Conditioning on a mode of ON before we reach home, thus, it makes our life convenient, simpler with saved time, energy and money.

2. Wearable: These applications mounted with software and sensors that collect data about consumers and afterward pre-processed to extract needed insights about customers. The main determination of these devices is largely covering fitness, entertainment and health desires.

3. Connecting Cars: The vehicle is used for connecting car applications that are able to enhance its own processes like maintenance, the comfort of passengers using sensors and internet connectivity.

4. Industrial Internet: The upcoming application like Industrial Internet is the recent ping in the Industrial Sector. It is called as Industrial Internet of Things (IIoT). Industrial Internet application affords tracking tools, great probable for quality control and sustainability. Also used for real-time information exchanging determination.

\section{CONCLUSION}

This review paper introduced recent research activities in the elaboration of the web architecture into suppressed environment using embedded web services by using elemental REST architecture in this domain. The principle vital challenges for such a type of network are the challenges are routing, scalability, naming, identification, security, and privacy. The home automation usage Internet of Things (IoT), the domestic automation, which handling IOT has been empirically unwavering with its function and give contentment while linking sober appliances. This instrumentation easily governs by means of internet. The designed system not only monitors the sensor data, but also actuates a process according to the demand. The sensor data like temperature, light, gas, motion sensors. The proposed outgrowth of the project objectives as multiple benefits, eliminating human involvement, reduces manpower, humanitarian efforts, which are frequently required to manually toggle the lights, motor and any type of electrical device ON/OFF and saving on electricity bills of the university or any other organization it is deployed. The most importantly, protect valuable natural resources by reducing electrical energy consumption.

\section{REFERENCES}

w[1] X. L. X. Z. C. Y. Jian Lia, "Automatic Control System of Intelligent Building Based on WebAccess," in World Congress on Automatic Control and Automation Conference, Chingqing, 2008.

[2] A. S. A. S. K. N. Sahana M N, "Home energy management leveraging open IoT protocol stack," in Recent 
Advance in intelligent Computational Systems Conference, Trivandrum, 2015.

[3] M. A. A. P. a. M. I. A. J. Whittle, "SENSOR NETWORKS FOR MONITORING AND CONTROL OF WATER DISTRIBUTION SYSTEMS," in International Conference on Structural Health Monitoring of Intelligent Infrastructure, Hong Kong, 2013.

[4] M. I. A. D. M. C. O. a. N. D. Xenofon Fafoutis, "Energy-Efficient Medium Access Control for Energy Harvesting Communications.," in IEEE Transaction on Consumer Electronics Conference, 2015.

[5] L. J.-p. L. L. Z. W. Liu Xue-Feng, "The Design and Application of the Optimize Control Management system to the Central Air-Conditioning Based On Intelligent Building Technology," in State Key Laboratory of Subtropical Building Science Conference, South China, 2010.

[6] M. A. J. M. I. J. Z. J. a. Y. L. S. A. Vaghefi, "A Hybrid Physics-Based and Data Driven Approach to Optimal Control of Building Cooling/Heating Systems," in IEEE TRANSACTION ON AUTOMATION SCIENCE AND ENGINEERING CONFERENCE, Piscataway, 2014.

[7] R. B. Pavithra. D, "IoT based Monitoring and Control System for Home Automation," in Global Conference on Communication Technologies, 2015.

[8] S. M. I. S. C. M. F. I. S. D. T. K. a. S. P. S. G. Nagender Kumar Suryadevara, "WSN-Based Smart Sensors and Actuator for Power Management in Intelligent Buildings.," in IEEE/ASME TRANSACTIONS ON MECHATRONICS , 2014.

[9] M. I. I. B. M. I. a. R. S. S. F. I. Prasenjit Chanak, "Simultaneous Mobile Sink Allocation in Home Environments with Applications in Mobile Consumer Robotics.," in IEEE Transaction on Consumer electronics Conference, India, 2015.

[10] S. M. I. A. M. M. M. R. a. W. U. M. I. Maria Pia Fanti, "A District Energy Management Based on Thermal Comfort Satisfaction and Real-Time Power Balancing.," in IEEE Transaction on Automation and Engineering, Automation and Engineering, 2015.

[11] D. L. J. B. a. K. S. Dongyu Wang, "AnyControl- IoT based Home Appliances Monitoring and Controlling," in IEEE 39th Annual International Computers, Software \& Applications Conference, Japan, 2015.

[12] P. G. J. C. Anisha Gupta, "IoT based Power Efficient System Design using Automation for Classrooms," in Third International Conference on Image Processing, Himachal Pradesh,India, 2015.

[13] n.-W. K. B.-T. L. C. H. C. a. J. W. C. Seung-Chul Son, "A Time Synchronization Technique for CoAP-based Home Automation Systems," in IEEE transactions on Consumer Electronics Conference, Korea, 2016.

[14] S. L. Zach Shelby, "EMBEDDED WEB SERVICES," in IEEE Wireless Communications Conference, Finland, 2010. 